\title{
On Probabilistic Averaging in Bounded Commutative Residuated $\ell$-monoids
}

\author{
Hongjun Zhou ${ }^{1}$ Guojun Wang ${ }^{1,2}$ \\ ${ }^{1}$ Institute of Mathematics, Shaanxi Normal University, Xi'an 710062, China \\ ${ }^{2}$ Research Center for Science, Xi'an Jiaotong University, Xi'an 710049, China
}

\begin{abstract}
Bounded commutative $R \ell$-monoids are a generalization of MV-algebras as well as of BL-algebras. With the probability measure on the set of states on bounded commutative $\ell$-monoids, we introduce the satisfiability degrees of propositions as their average truth degrees. If we consider the probability measure only on the set of state-morphisms, the concept of satisfiability degrees will naturally induce a similarity degree between two propositions of any bounded commutative $R \ell$-monoid. Then we can define a pseudo-metric on a bounded commutative $R \ell$-monoid and study the properties of such a metric space. Our result is an algebraic counterpart as well as a generalization of the integrated semantics for fuzzy logic.
\end{abstract}

Keywords: Bounded commutative $R \ell$-monoid, State; State-morphism, Probability measure, Satisfiability degree, $\mathrm{BCR} \ell$ metric space

\section{Introduction}

States as the average truth degrees of propositions in Łukasiewicz logic were introduced firstly on MValgebras by Mundici [10] roughly 40 years after introducing MV-algebras by Chang [1] as an algebraic counterpart of Łukasiewicz infinite-valued propositional logic. Because MV-algebras, $M$, are intervals in unital lattice ordered groups ( $\ell$-groups), $(G, u)$, due to Mundici's representation theorem [9], $M=\Gamma(G, u)=\{g \in G: 0 \leq g \leq u\}$, where $u$ is a strong order unit, states on $M V$ algebras can be defined as the restriction of normalized positive group-homomorphisms to the intervals $M=\Gamma(G, u)$. This means that on any MV-algebra we have a partial addition "+". BLalgebras have been introduced by Hájek [7] as algebras of basic fuzzy logic containing all logics behind fuzzy reasoning as special cases. Hence BLalgebras are also a generalization of MV-algebras, however, BL-algebras admit no partial addition.
The same problem arises also for bounded commutative $R \ell$-monoids introduced by Dvurečenskij [3] as a generalization of BL-algebras. Commutative $R \ell$-monoids are duals to commutative dually residuated lattice ordered monoids (DR $\ell$-monoids) which were introduced by Swamy [15] as a common generalization of Brouwerian algebras and abelian -groups.

Luckily, Georgescu [6] has successfully introduced the states (called Bosbach states there) on BL-algebras which in case of MV-algebras coincide with the notion of states for MV-algebra. Inspired by Georgescu, Dvurečenskij [3] recently introduced also the states on bounded commutative $\mathrm{R} \ell$-monoids and proved that every bounded commutative $\mathrm{R} \ell$-monoid admits at least a state. It may happen that some bounded commutative $\mathrm{R} \ell$ monoid admits two more states, for instance, $s_{1}$ and $s_{2}$, this gives rise to a question: which of $s_{1}(x)$ and $s_{2}(x)$ should be taken as the average truth degree of the proposition $x$ ? Does there exist an effective method to average all the truth degrees $s(x)$ ? The aim of the present paper is to give a positive answer to the question above. The idea behind our calculus is very simple, for the case that the set $\mathcal{S}(M)$ of states on $M$ is finite, suppose that $\mathcal{S}(M)=\left\{s_{1}, \cdots, s_{n}\right\}$ and the set of truth degrees of the proposition $x$ is $\left\{s_{i}(x) \mid i=1, \cdots, n\right\}=$ $\left\{\alpha_{1}, \cdots, \alpha_{m}\right\}$, then the probability of $x$ taking the truth degree $\alpha_{j}$ is equal to $\frac{k_{j}}{n}$ where $k_{j}$ is the number of states whose value at $x$ is $\alpha_{j}$. This suggests that the average truth degree should be $\sum_{j=1}^{m} \alpha_{j} \cdot \frac{k_{j}}{n}$. Following this idea, we shall consider the general case later in the present paper.

The rest of the present paper is structured as follows: In order to make the paper as selfcontained as possible, we shall recall in section 2 the representations of bounded commutative $\mathrm{R} \ell$ monoids and review some basic notions and concepts such as states and state-morphisms that shall be used throughout the paper. In section 3 , as a generalization of the basic idea mentioned 
above, we firstly consider the probability measure $\mu$ on a nonvoid subset $\Omega$ of the set of all states on a bounded commutative $\mathrm{R} \ell$-monoid, and introduce the concept of $(\Omega, \mu)$-satisfiability degrees of propositions which is determined by $\Omega$ and $\mu$. Such a generalization is interesting since the $(\{s\}, \mu)$-satisfiability degree of a proposition $x$ is just $s(x)$ for any state $s$. In section 4 , we consider the case that $\Omega$ is set of all state-morphisms on a bounded commutative $\mathrm{R} \ell$-monoid $M$. The $(\Omega, \mu)$-satisfiability degree of the distance function $d(x, y)=(x \rightarrow y) \wedge(y \rightarrow x)$ between two propositions $x$ and $y$ defined on $M$ will naturally induce a similarity measure between $x$ and $y$. Then we can define a pseudo-metric on $M$ and study the properties of this metric space. As an application, we give in section 5 an example for Lukasiewicz Lindenbaum algebra which shows that our result can be viewed as an algebraic counterpart of the integrated semantics proposed in [17] as well as a non-trivial generalization of [17]. Section 6 is some conclusion remarks.

\section{Preliminaries}

Commutative DR $\ell$-monoids were introduced by Swamy in [15] as a common generalization of commutative lattice ordered groups and Brouwerian algebras. Recently, it was shown in $[11,12,13]$ that also algebras of logics behind fuzzy reasoning, namely MV-algebras and duals of BL-algebras, can be regarded as particular cases of bounded commutative DR $\ell$-monoids. In the paper we deal with states on more general algebras than BL-algebras, hence we use the duals of DR $\ell$-monoids called $\mathrm{R} \ell$ monoids [3].

A commutative $\mathrm{R} \ell$-monoids is an algebra $M=$ $(M ; \odot, \vee, \wedge, \rightarrow, 1)$ of type $\langle 2,2,2,2,0\rangle$ satisfying the following conditions:

(i) $(M ; \odot, 1)$ is a commutative monoid,

(ii) $(M ; \vee, \wedge)$ is a lattice,

(iii) $x \odot y \leq z$ if and only if $x \leq y \rightarrow z$, for any $x, y, z \in M$,

(iv) $M$ satisfies the identity $((x \rightarrow y) \wedge 1) \odot x=$ $x \wedge y$.

By [15], commutative $\mathrm{R} \ell$-monoids form a variety of algebras of the indicated type. It is wellknown that a commutative $\mathrm{R} \ell$-monoid is bounded if and only if it is lower bounded. In such a case, 1 is the greatest element in $M$ and the identity (iv) is of the form $(x \rightarrow y) \odot x=x \wedge y$. Let us denote by 0 the smallest element in the bounded $\mathrm{R} \ell$-monoid $M$ and consider such $\mathrm{R} \ell$-monoids, called bounded commutative $\mathrm{R} \ell$-monoids, as algebras $M=(M ; \odot, \vee, \wedge, \rightarrow$ $, 0,1)$ of type $\langle 2,2,2,2,0,0\rangle$. Moreover, it is easy to verify that every bounded commutative $\mathrm{R} \ell$-monoid is a distributive lattice.

By [13], BL-algebras (and hence also MValgebras) are special cases of bounded commutative $\mathrm{R} \ell$-monoids. Namely, the class of BL-algebras is the variety of bounded $\mathrm{R} \ell$-monoids defined by the identity $(x \rightarrow y) \vee(y \rightarrow x)=1$, and this means by [16] that BL-algebras are exactly the bounded $\mathrm{R} \ell$-monoids which are representable as subdirect products of linearly ordered $\mathrm{R} \ell$-monoids. On the other hand, the class of bounded commutative $\mathrm{R} \ell$-monoids is essentially larger than that of BL-algebras (see Example 4.13 in [3]).

Define on any bounded commutative $\mathrm{R} \ell$ monoid $M$ the unary operation ' $: M \rightarrow M$ such that $x^{\prime}=x \rightarrow 0$ for each $x \in M$. Then, by [11, 12], $M$ is an MV-algebra if and only if $M$ satisfies the identity $x^{\prime \prime}=x$, for all $x \in M$.

One can consider on any $\mathrm{R} \ell$-monoid $M$ the distance function $d(x, y)=(x \rightarrow y) \wedge(y \rightarrow x)$, for each $x, y \in M$.

Proposition 2.1 (Swamy [15], Rachůnek and Slezák [14]). In any bounded commutative $\mathrm{R} \ell$-monoid $M$ we have

(1) $x \leq y$ if and only if $x \rightarrow y=1$,

(2) $x \rightarrow(y \wedge z)=(x \rightarrow y) \wedge(x \rightarrow z)$,

(3) $x \rightarrow(y \rightarrow z)=x \odot y \rightarrow z$,

(4) $d(x, y)=(x \vee y) \rightarrow(x \wedge y)$.

(5) $x \leq x^{\prime \prime}, x^{\prime}=x^{\prime \prime \prime}$,

(6) $x \odot(y \vee z)=(x \odot y) \vee(x \odot z)$,

(7) $x \odot(y \wedge z)=(x \odot y) \wedge(x \odot z)$.

Let $M$ be a bounded commutative $\mathrm{R} \ell$-monoid, then a mapping $s: M \rightarrow[0,1]$ is said to be a state if for all $x, y \in M$,

(i) $s(x)+s(x \rightarrow y)=s(y)+s(y \rightarrow x)$,

(ii) $s(0)=0$ and $s(1)=1$.

It is easy to verify that if $M$ is an MV-algebra, then a mapping $s: M \rightarrow[0,1]$ is a state if and only if (i) $s(1)=1$, and (ii) $s(x \oplus y)=s(x)+s(y)$ whenever $x \odot y=0$, where $x \oplus y=x^{\prime} \rightarrow y$.

We denote by $\mathcal{S}(M)$ the set of all states on $M$. It is interesting to ask whether $\mathcal{S}(M) \neq \emptyset$. The authors of [3] has shown that $\mathcal{S}(M)$ is a nonempty compact convex Hausdorff space.

Proposition 2.2(Dvurečenskij and Rachůnek [3]). Let $s$ be a state on a bounded commutative $\mathrm{R} \ell$-monoid $M$. Then for any $x, y \in M$,

(1) $s\left(x^{\prime}\right)=1-s(x)$, 
(2) $s\left(x^{\prime \prime}\right)=s(x)$,

(3) if $x \leq y$, then $1+s(x)=s(y)+s(y \rightarrow x)$,

(4) if $x \leq y$, then $s(x) \leq s(y)$,

(5) $s(x \odot y)=1-s\left(x \rightarrow y^{\prime}\right)$,

(6) $s(x)+s(y)=s(x \odot y)+s\left(y^{\prime} \rightarrow x\right)$,

(7) $s(d(x, y)) \leq s(d(x \rightarrow y, y \rightarrow x))$,

(8) $s\left(d\left(x^{\prime}, y^{\prime}\right)\right)=s(d(x, y))$,

(9) $s\left(x^{\prime} \rightarrow y^{\prime}\right)=1+s(x)-s(x \vee y)$,

(10) $s\left(x^{\prime} \rightarrow y^{\prime}\right)=s\left(y^{\prime \prime} \rightarrow x^{\prime \prime}\right)$,

(11) $s(x)+s(y)=s(x \vee y)+s(x \wedge y)$,

(12) if $x \vee y=1$, then $1+s(x \wedge y)=s(x)+s(y)$.

The standard unit interval $[0,1]$ equipped with the operations: $x \odot y=(x+y-1) \vee 0, x \rightarrow$ $y=(1-x+y) \wedge 1, x \vee y=\max \{x, y\}$ and $x \wedge y=\min \{x, y\}, x, y \in M$, becomes an MValgebra, called the standard MV-algebra.

A mapping $s$ from $M$ into the standard MValgebra $[0,1]$ is said to be a state-morphism if, for all $x, y \in M$ we have
(i) $s(x \rightarrow y)=s(x) \rightarrow s(y)$,
(ii) $s(x \wedge y)=\min \{s(x), s(y)\}$,
(iii) $s(1)=1$,

It is straightforward that every state-morphism on $M$ is a state on $M$.

We denote by $\mathcal{S M}(M)$ the system of all statemorphisms of $M$. The authors of [3] have also shown that there is a one-to-one correspondence between $\mathcal{S} \mathcal{M}(M)$ and $\mathcal{M F}(M)$ of maximal filters of $M$. Due to Zorn's lemma, $\mathcal{M F}(M)$ is nonempty, hence $\mathcal{S} \mathcal{M}(M) \neq \emptyset$. For the details of filters of bounded commutative $\mathrm{R} \ell$-monoids, we refer to [3].

\section{3. $(\Omega, \mu)$-satisfiability degrees of propositions}

Let $M$ be a sounded commutative $\mathrm{R} \ell$-monoid, $\Omega$ be a nonempty subset of $\mathcal{S}(M)$. Note that $\emptyset \neq$ $\mathcal{S} \mathcal{M}(M) \subseteq \mathcal{S}(M)$.

Suppose that $\mathcal{A}$ is a $\sigma$-algebra on $\Omega, \mu$ is the probability measure on $\Omega$, then $(\Omega, \mathcal{A}, \mu)$ becomes a probability measure space [8]. $\forall x \in M$, define $\bar{x}: \Omega \rightarrow[0,1]$ by $\bar{x}(x)=s(x), s \in \Omega$, then $\bar{x}$ is a $\mu$-integrable function on $\Omega$ for every $x$ of $M$.

Definition 3.1. Let $(\Omega, \mathcal{A}, \mu)$ be a probability measure space. $\forall x \in M$, then the integral

$$
\tau_{\Omega, \mu}(x)=\int_{\Omega} \bar{x}(s) d \mu
$$

is called the $(\Omega, \mu)$-satisfiability degree of the proposition $x$. The subscripts $\Omega$ and $\mu$ will be omitted if $\Omega=\mathcal{S M}(M)$ and $\mu$ can be determined from the context.

By the definition of integral, it is obvious that, if $\Omega=\left\{s_{1}, \cdots, s_{n}\right\}$ is finite, and $\mu$ is the evenly distributed probability measure on $\Omega$, then

$$
\tau_{\Omega, \mu}(x)=\sum_{j=1}^{m} \alpha_{j} \mu\left(\Sigma\left(x, \alpha_{j}\right)\right)=\sum_{j=1}^{m} \alpha_{j} \cdot \frac{\left|\Sigma\left(x, \alpha_{j}\right)\right|}{n},
$$

where $\Sigma\left(x, \alpha_{j}\right)=\left\{s \in \Omega \mid s(x)=\alpha_{j}\right\}, \alpha_{j} \in \Omega(x)=$ $\left\{s_{1}(x), \cdots, s_{n}(x)\right\}=\left\{\alpha_{1}, \cdots, \alpha_{m}\right\}$. In particular, if $\Omega$ is a singleton $\{s\}$, then $\tau_{\Omega, \mu}(x)=\bar{x}(s) \mu(\Omega)=$ $s(x)$ even if $\mu$ is not evenly distributed.

Proposition 3.2. $\tau_{\Omega, \mu}$ has the following properties for $x, y \in M$.

(1) $0 \leq \tau_{\Omega, \mu}(x) \leq 1$,

(2) $\tau_{\Omega, \mu}(0)=0, \tau_{\Omega, \mu}(1)=1$,

(3) $\tau_{\Omega, \mu}\left(x^{\prime}\right)=1-\tau_{\Omega, \mu}(x)$,

(4) $\tau_{\Omega, \mu}\left(x^{\prime \prime}\right)=\tau_{\Omega, \mu}(x)$,

(5) if $x \leq y$, then $\tau_{\Omega, \mu}(x) \leq \tau_{\Omega, \mu}(y)$,

(6) $\tau_{\Omega, \mu}(x \odot y)=1-\tau_{\Omega, \mu}\left(x \rightarrow y^{\prime}\right)$,

(7) $\tau_{\Omega, \mu}\left(d\left(x^{\prime}, y^{\prime}\right)\right)=\tau_{\Omega, \mu}(d(x, y))$,

(8) $\tau_{\Omega, \mu}\left(x^{\prime} \rightarrow y^{\prime}\right)=\tau_{\Omega, \mu}\left(y^{\prime \prime} \rightarrow x^{\prime \prime}\right)$,

(9) $\tau_{\Omega, \mu}(x)+\tau_{\Omega, \mu}(y)=\tau_{\Omega, \mu}(x \vee y)+\tau_{\Omega, \mu}(x \wedge y)$, $x)$.

(10) $\tau_{\Omega, \mu}(x)+\tau_{\Omega, \mu}(x \rightarrow y)=\tau_{\Omega, \mu}(y)+\tau_{\Omega, \mu}(y \rightarrow$

Proof. From Proposition 2.2, the proof is straightforward. Take the item (10) as an example. $\forall s \in \Omega$,

$$
\begin{aligned}
(\bar{x}+\overline{x \rightarrow y})(s) & =\bar{x}(s)+\overline{x \rightarrow y}(s) \\
& =s(x)+s(x \rightarrow y) \\
& =s(y)+s(y \rightarrow x) \\
& =\bar{y}(s)+\overline{y \rightarrow x}(s) \\
& =(\bar{y}+\overline{y \rightarrow x})(x),
\end{aligned}
$$

then we have

$$
\begin{aligned}
& \tau_{\Omega, \mu}(x)+\tau_{\Omega, \mu}(x \rightarrow y) \\
= & \int_{\Omega} \bar{x}(s) d \mu+\int_{\Omega} \overline{x \rightarrow y}(s) d \mu \\
= & \int_{\Omega}(\bar{x}+\overline{x \rightarrow y})(s) d \mu \\
= & \int_{\Omega}(\bar{y}+\overline{y \rightarrow x})(s) d \mu \\
= & \int_{\Omega} \bar{y}(s) d \mu+\int_{\Omega} \overline{y \rightarrow x}(s) d \mu \\
= & \tau_{\Omega, \mu}(y)+\tau_{\Omega, \mu}(y \rightarrow x) .
\end{aligned}
$$

It follows from the items (2) and (10) of Proposition 3.2 that $\tau_{\Omega, \mu}$ is also a state on $M$. However, it does not need to be a state-morphism on $M$, please see the following example.

Example 3.3. (i) Let $M$ be the diamond lattice, i.e., $M=\{0, a, b, 1\}, 0^{\prime}=1,1^{\prime}=0, a^{\prime}=b, b^{\prime}=$ $a, a \vee b=1, a \wedge b=0$, then $M$ is a Boolean algebra. 
If define $x \odot y=x \wedge y, x \rightarrow y=x^{\prime} \vee y, x, y \in M$, then $M$ is also an MV-algebra. It is not difficult to verify that $\mathcal{S}(M)=\{s: M \rightarrow[0,1] \mid$ $s(0)=0, s(1)=1, s(a)+s(b)=1\}$, and $\mathcal{S} \mathcal{M}(M)=$ $\left\{s_{1}, s_{2}\right\}$ where $s_{1}(a)=s_{2}(b)=1, s_{1}(b)=s_{2}(a)=$ $0, s_{i}(0)=0, s_{i}(1)=1, i=1,2$. Clearly, there is a one-to-one correspondence between $\mathcal{S}(M)$ and $[0,1]$, hence we can identify $\mathcal{S}(M)$ with $[0,1]$. Let $\mu_{1}$ be the Lebesgue measure on $\mathcal{S}(M)$, then $\tau_{\mathcal{S}(M), \mu_{1}}(a)=\int_{\mathcal{S}(M)} \bar{a}(s) d \mu_{1}=\int_{[0,1]} x d x=\frac{1}{2}$, and similarly, $\tau_{\mathcal{S}(M), \mu_{1}}(b)=\frac{1}{2}$. Note that $\tau_{\mathcal{S}(M), \mu_{1}} \in$ $\mathcal{S}(M)$. Let $\mu_{2}$ be the evenly distributed probability measure on $\mathcal{S} \mathcal{M}(M)$, then $\tau_{\mathcal{S} \mathcal{M}(M), \mu_{2}}(a)=$ $0 \times \mu\left(\left\{s_{2}\right\}\right)+1 \times \mu\left(\left\{s_{1}\right\}\right)=\frac{1}{2}=\tau_{\mathcal{S M}(M), \mu_{2}}(b)$. We also have $\tau_{\mathcal{S M}(M)}, \mu_{2} \in \mathcal{S}(M)$, but $\tau_{\mathcal{S M}(M)}, \mu_{2} \notin$ $\mathcal{S} \mathcal{M}(M)$ even though each member of $\mathcal{S} \mathcal{M}(M)$ is a state-morphism since $\tau_{\mathcal{S M}(M)}, \mu_{2}$ does not commute with min-conjunction: $\tau_{\mathcal{S M}(M), \mu_{2}}(a \wedge b)=$ $\tau_{\mathcal{S M}(M), \mu_{2}}(0)=0$.

(ii) We recall that for every linearly ordered bounded commutative $\mathrm{R} \ell$-monoid $M, \mathcal{S}(M)$ as a singleton coincides with $\mathcal{S} \mathcal{M}(M)$. Let $M$ be the standard MV-algebra and $\mathcal{S}(M)=\{s\}$, then $\tau_{\mathcal{S}(M), \mu}(x)=s(x)=x, x \in[0,1]$.

(iii) As an application, we will give in section 5 a third example for Łukasiewicz Lendinbaum algebra which shows that the result of the present paper can be viewed as an algebraic counterpart of integrated semantics for fuzzy logic proposed in [17] as well as a generalization of [17].

$\tau$ has other interesting properties besides those given in Proposition 3.2, which correspond to the formal inference rules such as MP and HS in Łukasiewicz infinite-valued propositional logic.

Proposition 3.4. Let $x, y \in M, \alpha, \beta \in[0,1]$. If $\tau(x) \geq \alpha, \tau(x \rightarrow y) \geq \beta$, then $\tau(y) \geq \alpha+\beta-1$.

Proof. Consider firstly the standard MValgebra $[0,1]$, it is easy to check that

$$
a+(a \rightarrow b)-1 \leq b, \quad a, b \in[0,1]
$$

Note that $a \odot b=(a+b-1) \vee 0$ and $a \odot(a \rightarrow b) \leq b$, then the preceding inequality automatically holds.

$$
\begin{aligned}
\forall x, y \in M, \forall s \in \Omega & =\mathcal{S M}(M), \\
(\bar{x}+\overline{x \rightarrow y}-\overline{1})(s) & =\bar{x}(s)+\overline{x \rightarrow y}(s)-1 \\
& =s(x)+s(x \rightarrow y)-1 \\
& =s(x)+(s(x) \rightarrow s(y))-1 \\
& \leq s(y)=\bar{y}(s),
\end{aligned}
$$

then

$$
\begin{aligned}
\tau(y) & =\int_{\Omega} \bar{y} d \mu \\
& \geq \int_{\Omega}(\bar{x}+\overline{x \rightarrow y}-\overline{1})(s) d \mu \\
& =\int_{\Omega} \bar{x}(s) d \mu+\int_{\Omega} \overline{x \rightarrow y}(s) d \mu-1 \\
& =\tau(x)+\tau(x \rightarrow y)-1 \\
& \geq \alpha+\beta-1 .
\end{aligned}
$$

Corollary 3.5. (i) If $\tau(x)=\tau(x \rightarrow y)=1$, then $\tau(y)=1$,

(ii) If $\tau(x \rightarrow y) \geq \alpha, \tau(y \rightarrow z) \geq \beta$, then $\tau(x \rightarrow$ $z) \geq \alpha+\beta-1$.

Proof. (i) It follows from Proposition 3.4 that $\tau(y) \geq \tau(x)+\tau(x \rightarrow y)-1=1+1-1=1$, hence $\tau(y)=1$.

(ii) We also note that $\overline{x \rightarrow y}=\bar{x} \rightarrow \bar{y}$ on $\Omega=$ $\mathcal{S} \mathcal{M}(M)$. Then $\forall s \in \Omega$,

$$
\begin{aligned}
& \overline{x \rightarrow y}(s)=s(x \rightarrow y) \\
& =s(x) \rightarrow s(y) \\
& \leq(s(y) \rightarrow s(z)) \rightarrow(s(x) \rightarrow s(z)) \\
& =\overline{y \rightarrow z}(s) \rightarrow \overline{x \rightarrow z}(s) \\
& =\overline{(y \rightarrow z) \rightarrow(x \rightarrow z)}(s) \text {, }
\end{aligned}
$$

thus $\tau(x \rightarrow y) \leq \tau((y \rightarrow z) \rightarrow(x \rightarrow z))$.

Now, from (i) we have

$$
\begin{aligned}
\tau(x \rightarrow z) & \geq \tau(y \rightarrow z)+\tau((y \rightarrow z) \rightarrow(x \rightarrow z))-1 \\
& \geq \tau(y \rightarrow z)+\tau(x \rightarrow y)-1 \\
& \geq \alpha+\beta-1
\end{aligned}
$$

Proposition 3.6. Let $x, y, z \in M, 0 \leq \varepsilon<1$. If $\tau(x \rightarrow y) \geq 1-\varepsilon, \tau(x \rightarrow z) \geq 1-\varepsilon$, then

$$
\tau(x \rightarrow y \wedge z) \geq(1-\sqrt{2 \varepsilon})^{2},
$$

where we require that both $E_{1}=\{s \in \Omega$ | $\overline{x \rightarrow y}(s)<1-\sqrt{2 \varepsilon}\}$ and $E_{2}=\{s \in \Omega \mid \overline{x \rightarrow z}(s)<$ $1-\sqrt{2 \varepsilon}\}$ be $\mu$-measurable, i.e., $E_{1}, E_{2} \in \mathcal{A}$.

Proof. Let $d_{1}=\mu\left(E_{1}\right)$ and $d_{2}=\mu\left(E_{2}\right)$, then

$$
\begin{aligned}
\tau(x \rightarrow y) & =\int_{\Omega} \overline{x \rightarrow y}(s) d \mu \\
& =\int_{E_{1}} \overline{x \rightarrow y}(s) d \mu+\int_{\Omega-E_{1}} \overline{x \rightarrow y}(s) d \mu \\
& \leq d_{1}(1-\sqrt{2 \varepsilon})+1-d_{1} \\
& =1-d_{1} \sqrt{2 \varepsilon} .
\end{aligned}
$$

By assumption, $1-d_{1} \sqrt{2 \varepsilon} \geq 1-\varepsilon$, then we have $d_{1} \leq \sqrt{\frac{1}{2} \varepsilon}$. Similarly, $d_{2} \leq \sqrt{\frac{1}{2} \varepsilon}$. Moreover, on $\Omega-\left(E_{1} \cup E_{2}\right), \overline{x \rightarrow y}(s) \geq 1-\sqrt{2 \varepsilon}, \overline{x \rightarrow y}(s) \geq$ $1-\sqrt{2 \varepsilon}$, therefore

$$
\begin{aligned}
\overline{x \rightarrow(y \wedge z)}(s) & =\overline{(x \rightarrow y) \wedge(x \rightarrow z)}(s) \\
& =(\overline{x \rightarrow y} \wedge \overline{x \rightarrow z})(s) \\
& =\overline{x \rightarrow y}(s) \wedge \overline{x \rightarrow z}(s) \\
& \geq 1-\sqrt{2 \varepsilon} .
\end{aligned}
$$


On the other hand,

$$
\begin{aligned}
\mu\left(\Omega-\left(E_{1} \cup E_{2}\right)\right) & \geq \mu(\Omega)-\mu\left(E_{1}\right)-\mu\left(E_{2}\right) \\
& =1-d_{1}-d_{2} \\
& \geq 1-2 \sqrt{\frac{1}{2} \varepsilon} \\
& =1-\sqrt{2 \varepsilon} .
\end{aligned}
$$

Therefore

$$
\begin{aligned}
\tau(x \rightarrow y \wedge z) & \geq \int_{\Omega-\left(E_{1} \cup E_{2}\right)} \overline{x \rightarrow y \wedge z}(s) d \mu \\
& \geq(1-\sqrt{2 \varepsilon})^{2} .
\end{aligned}
$$

With $\tau$, we can define a similarity degree between two propositions.

Definition 3.7. Define $\eta: M \times M \rightarrow[0,1]$ as follows

$$
\eta(x, y)=\tau(d(x, y))=\tau((x \rightarrow y) \wedge(y \rightarrow x)),
$$

then $\eta$ is a similarity measure.

The reason for $\eta$ to be a similarity measure lies in the next proposition.

Proposition 3.8. $\eta$ has the following properties.

(1) $\eta(x, x)=1$,

(2) $\eta(x, y)=\eta(y, x)$

(3) $\eta\left(x^{\prime}, y^{\prime}\right)=\eta(x, y)$

(4) $\eta(x, z) \geq \eta(x, y)+\eta(y, z)-1$.

Proof. (1) and (2) are trivial. (3) follows from (7) of Proposition 3.2. We need only to prove (4).

In the standard MV-algebra $[0,1], a, b, c \in$ $[0,1]$, we have

$$
\begin{aligned}
& ((a \rightarrow b) \wedge(b \rightarrow a)+(b \rightarrow c) \wedge(c \rightarrow b)-1) \vee 0 \\
= & ((a \rightarrow b) \wedge(b \rightarrow a)) \odot((b \rightarrow c) \wedge(c \rightarrow b)) \\
= & (a \rightarrow b) \odot(b \rightarrow c) \wedge(a \rightarrow b) \odot(c \rightarrow b) \\
& \wedge(b \rightarrow a) \odot(b \rightarrow c) \wedge(b \rightarrow a) \odot(c \rightarrow b) \\
\leq \quad & (a \rightarrow b) \odot(b \rightarrow c) \wedge(c \rightarrow b) \odot(b \rightarrow a) \\
\leq \quad & (a \rightarrow c) \wedge(c \rightarrow a) .
\end{aligned}
$$

Then $\forall x, y, z \in M$, it is easy to check that

$$
\begin{aligned}
& \overline{(x \rightarrow z) \wedge(z \rightarrow x)}=(\bar{x} \rightarrow \bar{z}) \wedge(\bar{z} \rightarrow \bar{x}) \\
& \geq(\overline{(x)} \rightarrow \bar{y}) \wedge(\bar{y} \rightarrow \bar{x}) \\
& \begin{aligned}
= & \frac{+\bar{y} \rightarrow \bar{z}) \wedge(\bar{z} \rightarrow \bar{y})}{(x \rightarrow y) \wedge(y \rightarrow x)} \\
& +\overline{(y \rightarrow z) \wedge(z \rightarrow y)}-1
\end{aligned}
\end{aligned}
$$

and so

$$
\begin{aligned}
\eta(x, z)= & \int_{\Omega} \overline{(x \rightarrow z) \wedge(z \rightarrow x)}(s) d \mu \\
\geq & \int_{\Omega} \overline{(x \rightarrow y) \wedge(y \rightarrow x)}(s) d \mu \\
& +\int_{\Omega} \overline{(y \rightarrow z) \wedge(z \rightarrow y)}(s) d \mu-1 \\
= & \eta(x, y)+\eta(y, z)-1 .
\end{aligned}
$$

\section{BCR $\ell$ metric space}

Definition 4.1. Define $\rho: M \times M \rightarrow[0,1]$ as follows:

$$
\rho(x, y)=1-\eta(x, y) .
$$

Then, from Proposition 3.8, $\rho$ is a pseudo-metric on $M .(M, \rho)$ is said to be a BCR $\ell$ metric space.

Consider the set $[0,1]^{M}$ consisting of all functions $f: M \rightarrow[0,1]$. There are several ways to introduce topologies on $[0,1]^{M}$, e.g., the point wise convergence topology, the uniform convergence topology, and the compact topology, etc. (see[4]). One can introduce a metric on $[0,1]^{M}$ as well as follows, i.e., for $x, y \in M$, define

$$
\rho^{*}(x, y)=\int_{\Omega}|\bar{x}-\bar{y}| d \mu .
$$

It is obvious that $\rho^{*}: M \times M \rightarrow[0,1]$ is a pseudometric on $M$. An interesting question is: what is the relation between $\rho^{*}$ and $\rho$ ? the following theorem gives a positive answer, $\rho^{*}=\rho$.

Theorem 4.2. $\rho^{*}=\rho$.

Proof. $\forall x, y \in M, \forall s \in \Omega=\mathcal{S} \mathcal{M}(M)$,

$$
\begin{aligned}
& \overline{(x \rightarrow y) \wedge(y \rightarrow x)}(s) \\
= & ((\bar{x} \rightarrow \bar{y}) \wedge(\bar{y} \rightarrow \bar{x}))(s) \\
= & (s(x) \rightarrow s(y)) \wedge(s(y) \rightarrow s(x)) \\
= & (1-s(x)+s(y)) \wedge(1-s(y)+s(x)) \\
= & 1-|s(x)-s(y)| \\
= & 1-|\bar{x}-\bar{y}|(s),
\end{aligned}
$$

thus we have $\overline{1}-\overline{(x \rightarrow y) \wedge(y \rightarrow x)}=|\bar{x}-\bar{y}|$, and

$$
\begin{aligned}
\rho^{*}(x, y) & =\int_{\Omega}|\bar{x}-\bar{y}| d \mu \\
& =\int_{\Omega}(\overline{1}-\overline{(x \rightarrow y) \wedge(y \rightarrow x)})(s) d \mu \\
& =1-\int_{\Omega} \overline{(x \rightarrow y) \wedge(y \rightarrow x)}(s) d \mu \\
& =1-\eta(x, y) \\
& =\rho(x, y) .
\end{aligned}
$$

The proof is completed.

Note that the operation-preserving property of state-morphisms plays a crucial role in the proof of Theorem 4.2, hence Theorem 4.2 does not hold for $\Omega \nsubseteq \mathcal{S M}(M)$ in general.

In the sequel we shall show that all the operations ${ }^{\prime}, \odot, \vee, \wedge$ and $\rightarrow$ are all uniformly continuous with respect to $\rho$. To do so, we need a lemma.

Lemma 4.3. Let $x, y, u, v \in M, \alpha, \beta \in[0,1]$. If $\eta(x, u) \geq \alpha, \beta(y, v) \geq \beta$, then $\eta(x \rightarrow y, u \rightarrow v) \geq$ $\alpha+\beta-1$. 
Proof. In the standard MV-algebra $[0,1], a, b, c \in[0,1]$, we have

$$
a \rightarrow b \leq(b \rightarrow c) \rightarrow(a \rightarrow c),
$$

and similarly,

$$
b \rightarrow a \leq(a \rightarrow c) \rightarrow(b \rightarrow c) .
$$

Thus,

$$
\begin{aligned}
& (a \rightarrow b) \wedge(b \rightarrow a) \\
\leq \quad & ((b \rightarrow c) \rightarrow(a \rightarrow c)) \wedge((a \rightarrow c) \rightarrow(b \rightarrow c)) .
\end{aligned}
$$

Using the inequality above, we get that, $\forall x, y, u \in M$,

$$
\begin{aligned}
& (\bar{x} \rightarrow \bar{u}) \wedge(\bar{u} \rightarrow \bar{x}) \\
\leq \quad & ((\bar{u} \rightarrow \bar{y}) \rightarrow(\bar{x} \rightarrow \bar{y})) \wedge((\bar{x} \rightarrow \bar{y}) \rightarrow(\bar{u} \rightarrow \bar{y})) .
\end{aligned}
$$

It follows that

$$
\begin{aligned}
& \tau(((u \rightarrow y) \rightarrow(x \rightarrow y)) \wedge((x \rightarrow y) \rightarrow(u \rightarrow y))) \\
\geq \quad & \tau((x \rightarrow u) \wedge(u \rightarrow x))
\end{aligned}
$$

i.e.,

$$
\eta(x \rightarrow y, u \rightarrow y) \geq \eta(x, u) \geq \alpha .
$$

Similarly,

$$
\eta(u \rightarrow y, u \rightarrow v) \geq \eta(y, v) \geq \beta .
$$

By Proposition 3.8(4) we have

$$
\begin{aligned}
\eta(x \rightarrow y, u \rightarrow v) \geq & \eta(x \rightarrow y, u \rightarrow y) \\
& +\eta(u \rightarrow y, u \rightarrow v)-1 \\
\geq & \alpha+\beta-1 .
\end{aligned}
$$

Theorem 4.4. Let $(M, \rho)$ be a BCR $\ell$ metric space. Then the operations ${ }^{\prime}, \odot, \vee, \wedge$ and $\rightarrow$ are all uniformly continuous with respect to $\rho$.

Proof. It follows from Proposition 3.2(7) that

$$
\begin{aligned}
\rho\left(x^{\prime}, y^{\prime}\right) & =1-\tau\left(d\left(x^{\prime}, y^{\prime}\right)\right) \\
& =1-\tau(d(x, y)) \\
& =\rho(x, y) .
\end{aligned}
$$

This shows that' $: M \rightarrow M$ is uniformly continuous with respect to $\rho$.

Now, let $\rho(x, u) \leq \varepsilon, \rho(y, v) \leq \varepsilon$. Then $\eta(x, u) \geq 1-\varepsilon$ and $\eta(y, v) \geq 1-\varepsilon$, by Lemma 4.3 ,

$\eta(x \rightarrow y, u \rightarrow v) \geq(1-\varepsilon)+(1-\varepsilon)-1=1-2 \varepsilon$.

Therefore $\rho(x \rightarrow y, u \rightarrow v) \leq 2 \varepsilon$ which indicates the uniform continuity of $\rightarrow$.
Next, we turn to prove the uniform continuity of $\vee$. It is not difficult to check the following inequalities

$$
x \rightarrow u \leq x \vee y \rightarrow u \vee y,
$$

and

$$
u \rightarrow x \leq u \vee y \rightarrow x \vee y, \quad x, y, u \in M
$$

Then $\eta(x \vee y, u \vee y)=\tau((x \vee y \rightarrow u \vee y) \wedge(u \vee y \rightarrow$ $x \vee y)) \geq \tau((x \rightarrow u) \wedge(u \rightarrow x))=\eta(x, u)$. Similarly, we have $\eta(u \vee y, u \vee v) \geq \eta(y, v)$.

From Proposition 3.8(4), we get

$$
\begin{aligned}
\eta(x \vee y, u \vee v) \geq & \eta(x \vee y, u \vee y) \\
& +\eta(u \vee y, u \vee v)-1, \\
\geq & \eta(x, u)+\eta(y, v)-1 .
\end{aligned}
$$

which is equivalent to

$$
\rho(x \vee y, u \vee v) \leq \rho(x, u)+\rho(y, v) .
$$

Therefore $\vee$ is uniformly continuous with respect to $\rho$.

As analogues of the proof for $\vee$, we can prove the uniform continuities of $\wedge$ and $\odot$ respectively. It is left to the reader as an exercise.

The next theorem reveals the relationship between the metric completeness of $M$ as a metric space and the countable completeness of $M$ as a lattice.

Theorem 4.5. If the BCR $\ell$ metric space $(M, \rho)$ is metric complete, then $M$, as a lattice, is countable complete.

Proof. Let $(M, \rho)$ be a complete metric space, and $\Delta=\left\{x_{1}, x_{2}, \cdots\right\}$ be a subset of $M$. Put $y_{n}=\bigvee_{i=1}^{n} x_{i}$, then $y_{1}, y_{2}, \cdots$ is an increasing sequence. It follows from Proposition 3.2(5) that $\tau\left(y_{1}\right), \tau\left(y_{2}\right), \cdots$ is a non-decreasing sequence of the unit interval $[0,1]$, hence it is a Cauchy sequence. Observe that

$$
\overline{y_{n}} \rightarrow \overline{y_{m}}=1_{M}, \quad \overline{y_{m}} \rightarrow \overline{y_{n}}=\overline{1}-\overline{y_{m}}+\overline{y_{n}}
$$

whenever $n \leq m$. As a consequence, we have

$$
\begin{aligned}
& \rho\left(y_{m}, y_{n}\right) \\
= & 1-\eta\left(y_{m}, y_{n}\right) \\
= & 1-\tau\left(\left(y_{m} \rightarrow y_{n}\right) \wedge\left(y_{n} \rightarrow y_{m}\right)\right) \\
= & 1-\int_{\Omega}\left(\left(\overline{y_{m}} \rightarrow \overline{y_{n}}\right) \wedge\left(\overline{y_{n}} \rightarrow \overline{y_{m}}\right)\right)(s) d \mu \\
= & 1-\int_{\Omega}\left(\overline{y_{m}} \rightarrow \overline{y_{n}}\right)(s) d \mu \\
= & 1-\int_{\Omega}\left(\overline{1}-\overline{y_{m}}+\overline{y_{n}}\right)(s) d \mu \\
= & \int_{\Omega} \overline{y_{m}}(s) d \mu-\int_{\Omega} \overline{y_{n}}(s) d \mu \\
= & \tau\left(y_{m}\right)-\tau\left(y_{n}\right),
\end{aligned}
$$


which means that $y_{1}, y_{2}, \cdots$ is a Cauchy sequence in $(M, \rho)$. Let $y$ be the limit point of this sequence. Fix a number $n$, we have $y_{n}=y_{n} \wedge\left(y_{n+k}\right), k=$ $1,2, \cdots$. By the continuity of $\wedge$, the following holds:

$y_{n}=\lim _{k \rightarrow \infty}\left(y_{n} \wedge y_{n+k}\right)=y_{n} \wedge\left(\lim _{k \rightarrow \infty} y_{n+k}\right)=y_{n} \wedge y$.

Thus $y_{n} \leq y$, and $y$ is an upper bound of $\Sigma=$ $\left\{y_{1}, y_{2}, \cdots\right\}$. Suppose that $z$ is a upper bound of $\Sigma$, then

$y=\lim _{n \rightarrow \infty} y_{n}=\lim _{n \rightarrow \infty}\left(y_{n} \wedge z\right)=\left(\lim _{n \rightarrow \infty} y_{n}\right) \wedge z=y \wedge z$,

which entails that $y \leq z$. Up to now, we have shown that $y=\sup \Sigma \in M$. It is trivial that $\sup \Sigma=$ $\sup \Delta$. Similarly inf $\Delta \in M$. This concludes the proof.

\section{An application}

As an application, we give an example for Lukasiewicz Lindenbaum algebra which shows that the result of the paper can be viewed as an algebraic counterpart of the integrated semantics for fuzzy logic proposed in [17] as well as a generalization of [17]. We recall only the notions that shall be used later. For the representation of Łukasiewicz infinite-valued propositional logic, we refer to $[7,2,5]$.

The Eukasiewicz propositional calculus has the set $S$ of propositional variables $p_{1}, p_{2}, \cdots$, connectives $\&, \rightarrow$ and the truth constant $\overline{0}$ for 0 . The set $F(s)$ of all well-formed formulas is defined in the obvious way: each propositional variable is a formula; $\overline{0}$ is a formula; if $\varphi, \psi$ are formulas, then so are $\varphi \& \psi$ and $\varphi \rightarrow \psi$. Further connectives are defined as follows:

$$
\begin{aligned}
& \text { - } \varphi \wedge \psi \text { is } \varphi \&(\varphi \rightarrow \psi), \\
& \text { - } \varphi \vee \psi \text { is }(\varphi \rightarrow \psi) \rightarrow \psi, \\
& \text { - } \neg \psi \text { is } \varphi \rightarrow \overline{0} .
\end{aligned}
$$

The truth degree set of Łukasiewicz infinitevalued logic is the standard MV-algebra $[0,1]$. An evaluation of propositional variables is a mapping $e$ assigning to each propositional variable $p$ its truth degree $e(p) \in[0,1]$. This extends uniquely to the evaluation of all formulas as follows

$$
\begin{aligned}
& e(\overline{0})=0, \\
& e(\varphi \rightarrow \psi)=e(\varphi) \rightarrow e(\psi), \\
& e(\varphi \& \psi)=e(\varphi) \odot e(\psi) .
\end{aligned}
$$

The set of all evaluations is denoted by $\bar{\Omega}$.
Each formula $\varphi$ can induce a truth degree function, denoted by $\bar{\varphi}$, in a natural way: suppose that $\varphi$ is generated by the propositional variables $p_{1}, \cdots, p_{n}$, then the value of $\bar{\varphi}$ is, for each $n$-tuple $\left(x_{1}, \cdots, x_{n}\right)$ of truth degrees, given by

$$
\bar{\varphi}\left(x_{1}, \cdots, x_{n}\right)=e(\varphi)
$$

for any evaluation $e$ satisfying $e\left(p_{i}\right)=x_{i}$ for all $i=1, \cdots, n$.

The integral

$$
\tau^{*}(\varphi)=\int_{[0,1]^{n}} \bar{\varphi}\left(x_{1}, \cdots, x_{n}\right) d x_{1} \cdots d x_{n}
$$

is said to be the tautological degree of $\varphi[17]$.

In the sequel we shall prove that $\tau^{*}$ is just a special function defined from Łukasiewicz Lindenbaum algebra into the unit interval $[0,1]$ in the sense of Definition 3.1.

Two formulas $\varphi$ and $\psi$ are said to be logically equivalent, denote by $\varphi \approx \psi$, if $e(\varphi)=e(\psi)$ for any evaluation $e \in \bar{\Omega}$. It is routine to check that $\approx$ is a congruence with respect to the logical connectives $\neg, \&$ and $\rightarrow$. This suggests that the quotient algebra $F(S) / \approx$ with the induced operations $[\varphi] \odot[\psi]=[\varphi \& \psi]$ and $[\varphi] \rightarrow[\psi]=[\varphi \rightarrow \psi]$ becomes an MV-algebra.

Let $X_{n}=[0,1], \mu_{n}$ be the Lebesgue measure on $X_{n}$. Let $X=\prod_{n=1}^{\infty} X_{n}$ be the Cartesian product of $X_{n}^{\prime} s$, and $\mu$ be the infinite product of $\mu_{1}, \mu_{2}, \cdots$ [8].

Let $e \in \bar{\Omega}$, then $e$ is uniquely determined by its restriction $e \mid S$. Assume that $v\left(p_{k}\right)=e_{k}(k=$ $1,2, \cdots)$, then $\vec{e}=\left(e_{1}, e_{2}, \cdots\right) \in X$. Conversely, $\forall \vec{e}=\left(e_{1}, e_{2}, \cdots\right) \in X$, there exists a unique evaluation $e$ such that $e\left(p_{k}\right)=e_{k}(k=1,2, \cdots)$. That is to say, there is a one-to-one correspondence between $\bar{\Omega}$ and $X$, hence we can identify $\bar{\Omega}$ with $X$.

It is easy to verify that $\forall e \in \bar{\Omega}, e$ is a state morphism on $F(S) / \approx$. Define $\overline{[\varphi]}(e)=e(\varphi), e \in \bar{\Omega}$, then $\overline{[\varphi]}=\bar{\varphi}$ on $\bar{\Omega}$, and so

$$
\begin{aligned}
\tau_{\bar{\Omega}, \mu}([\varphi]) & =\int_{\bar{\Omega}} \overline{[\varphi]}(e) d \mu=\int_{X} \bar{\varphi}(e) d \mu \\
& =\int_{[0,1]^{n}} \bar{\varphi}\left(x_{1}, \cdots, x_{n}\right) d x_{1} \cdots d x_{n} \\
& =\tau^{*}(\varphi) .
\end{aligned}
$$

This shows that our result is a non-trivial generalization of [17]. Furthermore, the integrated semantics is established only from the logical point of view, while our result is established from the algebraic point of view, hance our result can be viewed as an algebraic counterpart of the integrated semantics for fuzzy logic. 


\section{Conclusions}

In the paper we have introduced the concept of satisfiability degrees of propositions in bounded commutative $\mathrm{R} \ell$-monoids with the intent of capturing the notion if average degree of truth of a proposition, which, to some extent, improves Mundici's theory. With this concept, we naturally introduced a pseudo metric on every bounded commutative $\mathrm{R} \ell$ monoid, and it is important that we proved all the operations are uniformly continuous with respect to this metric. The integrated semantics is a special case of our result, hence, our idea can be adapted to fuzzy logic to introduce the concept of tautological degrees of formulas with the parameter $\mu$ and to do graded reasoning as done in [18].

\section{References}

[1] C.C. Chang, Algebraic analysis of many valued logics, Trans, Amer. Math. Soc. 88(1958)467490.

[2] R. Cignoli, I.M.L. D’Ottaviano, D. Mundici, Algebraic Foundations of Many-Valued Reasoning, Kluwer Academic Publishers, Dordrecht, 2000.

[3] A. Dvurečenskij, J. Rachůnek, Probabilistic averaging in bounded commutative residuated $\ell$ monoids, Discrete Mathematics 306(2006)13171326.

[4] R. Engelking, General Topology, Heldermann Verlag, Berlin, 1989.

[5] S. Gottwald, A Treatise on Many-Valued Logics, Studies in Logic and Computation, Research Studies Press, Baldock, 2001.

[6] G. Georgescu, Bosbach states on fuzzy structures, Soft Comput 8(2004)217-230.

[7] P. Hájek, Metamathematics of Fuzzy logic, Kluwer Academic Publishers, Dordrecht, 1998.

[8] P.R. Halmos, Masure Theory, Springer-Verlag, New York, 1974.

[9] D. Mundici, Interpretation of AF $C^{*}$-algebras in Łukasiewicz sentential calculus, J. Funct. Anal. 65(1986)15-63.

[10] D. Mundici, Averaging the truth-value in Łukasiewicz logic, Studia Logic 55(1995)113127.
[11] J. Rachůnek, DR -semigroups and MValgebras, Czechoslovak Math. J. 48(1998) 365372.

[12] J. Rachůnek, MV-algebras are categorically equivalent to a class of $\mathrm{DR} \ell_{1(i)}$-semigroups, Math. Bohemica 123(1998) 437-441.

[13] J. Rachůnek, A duality between algebras of basic logic and bounded representable DR $\ell$ monoids, Math. Bochemica 126(2001)561-569.

[14] J. Rachůnek, J. Slezák, Negation in bonded commutative DR $\ell$-monoids, Czehoslovak Math. J., to appear.

[15] K.L.N. Swamy, Dually residuated lattice ordered semigroups, Math. Ann. 159(1965) 105114.

[16] K.L.N. Swamy, B.V. Subba Rao, Isometries in dually residuated lattice ordered semigrups, Math. Semin. Notes, Kobe Univ. 8(1980) 369380.

[17] G.J. Wang, Y. Leung, Integrated semantics and logic metric spaces, Fuzzy Sets and Systems 136(2003)71-91.

[18] H.J. Zhou, G.J.Wang, Generalized consistency degrees of theories w.r.t. formulas in several standard complete logic systems, Fuzzy Sets and Systems 157(2006)2058-2073. 